\author{
Rajat Jhamb*, Abhishek Juneja, SV \\ Madhu and S Giri \\ Department of Medicine, University College of \\ Medical Sciences and Guru Teg Bahadur Hospital, \\ Delhi, India
}

Dates: Received: 11 August, 2016; Accepted: 10 September, 2016; Published: 15 September, 2016

*Corresponding author: Rajat Jhamb, Associate Professor, Department of Medicine, University College of Medical Sciences and Guru Teg Bahadur Hospital, Delhi, India, Email: rajatjhamb@yahoo.com www.peertechz.com

Keywords: Obstructive jaundice, Tubercular lymphadenitis

\author{
Case Report
}

\section{Tubercular Lymphadenitis: As a Rare Cause of Obstructive Jaundice}

\section{Introduction}

Abdominal tuberculosis (TB) commonly affects the intestinal tract, lymph nodes, peritoneum and solid organs in varying combinations [1]. Obstructive jaundice secondary to abdominal TB is extremely rare and can be caused by TB enlargement of the head of the pancreas, TB lymphadenitis, TB stricture of the biliary tree, or a TB mass of the retroperitoneum [2]. We describe a patient with biliary obstruction caused by enlarged tubercular lymph nodes.

\section{Case Report}

A 42-year-old woman presented to our hospital with fever for 2 months and jaundice for 15 days with malaise and apparent weight loss over preceding 6 months. The total bilirubin was $6.7 \mathrm{mg} / \mathrm{dl}$ with conjugated hyperbilirubinemia of $4.9 \mathrm{mg} / \mathrm{dl}$; SGOT, SGPT were mildly raised with marked elevation of alkaline phosphatase. HBsAg, HIV and anti HCV antibodies were negative. Mantoux test was strongly positive with ESR of 86 . Chest X ray PA was normal (Figure 1). Abdominal ultrasonography revealed multiple necrotic lymph nodes in periportal and peripancreatic regions.

Contrast enhanced computed tomogram of the abdomen showed periportal, peripancreatic, gastrohepatic, and retroperitoneal lymphadenopathy suggesting abdominal Koch's (Figure 2)

Confluent lymph-nodal mass showing necrotic areas was seen in the hepatogastric, portal, peripancreatic, retropancreatic, portocaval region pushing the pancreas and portal vein anteriorly on magnetic resonance cholangiopancreatography suggestive of tubercular pathology. Mass is encasing and compressing the extrahepatic $\mathrm{CBD}$ just beyond the porta, beyond which $\mathrm{CBD}$ is not visualized with resultant proximal mild bilobular intrahepatic biliary radical dilatation. The mass measures approx. $8.3 * 5.8^{\star} 5.1 \mathrm{~cm}$ in its maximum dimension. Few discrete lymph nodes are also noted at porta, periaortic, postcaval and aortocaval region (Figure 3).

Percutaneous ultrasonography-guided fine needle aspiration of the confluent lymph nodal mass was performed and pathologic examination showed chronic granulomatous inflammation. AFB stain did not demonstrate acid-fast bacilli (Figure 4).

The patient was treated with anti-tubercular therapy. She regained her appetite and weight gradually increased within a month. Bilirubin level had decreased to 2.6 within 4 weeks.

\section{Discussion}

Hepatobiliary tuberculosis is extremely rare and difficult to diagnose [3]. The annual incidence of hepatobiliary TB is reported as $1.05 \%$ of all TB infections [4]. Abdominal tuberculosis causes obstructive jaundice by mechanical obstruction of the biliary tract either by compression from lymph nodes or mass lesion [5].

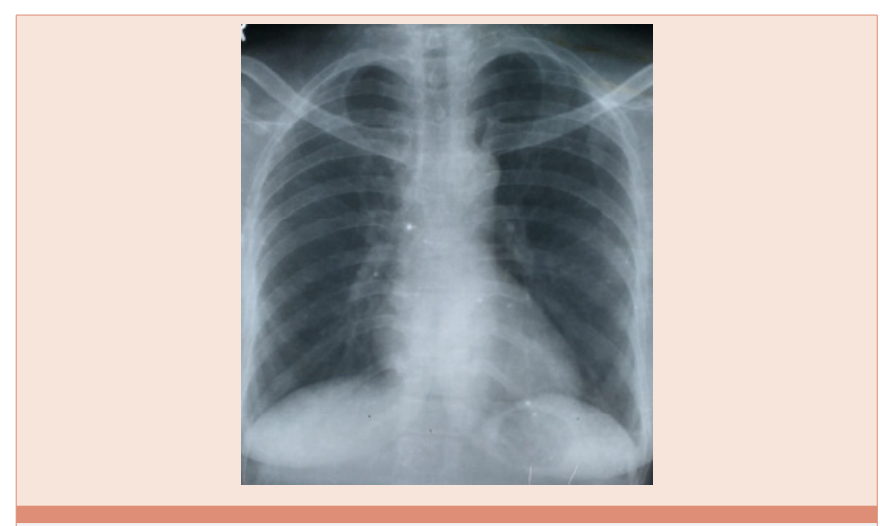

Figure 1: Chest Radiograph (PA view) 


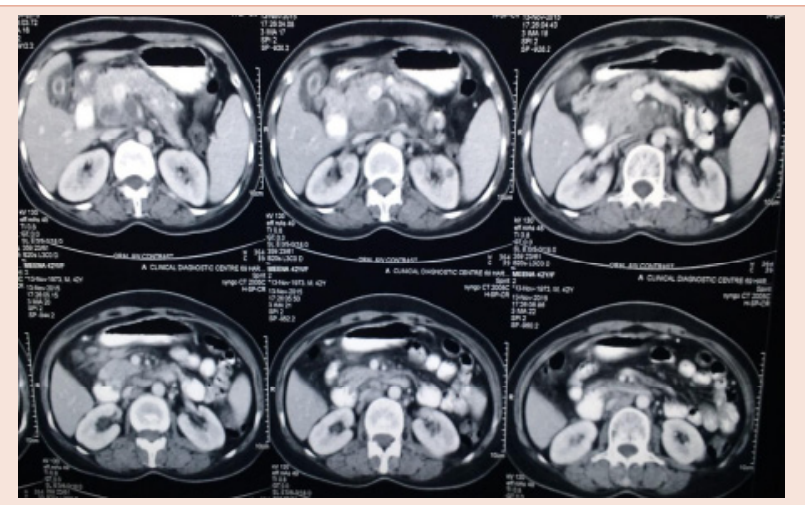

Figure 2: CECT abdomen showing necrotic lymph nodes.

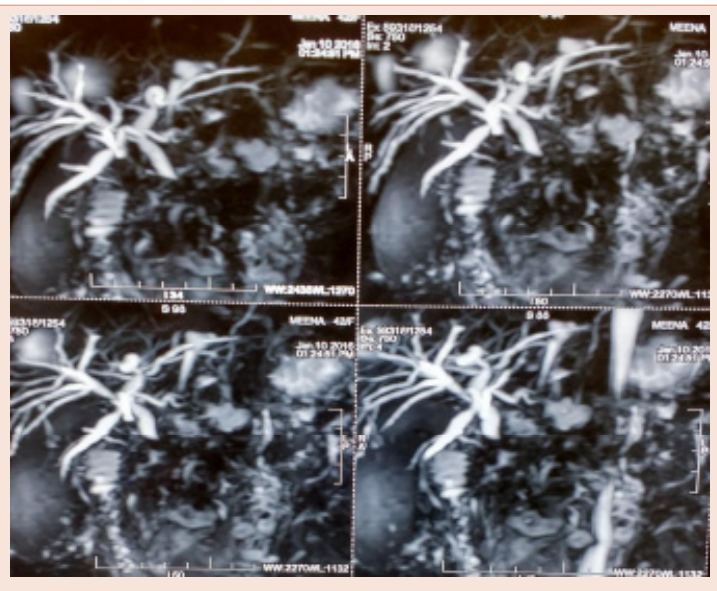

Figure 3: MRCP showing multiple lymph nodes with IHBRD

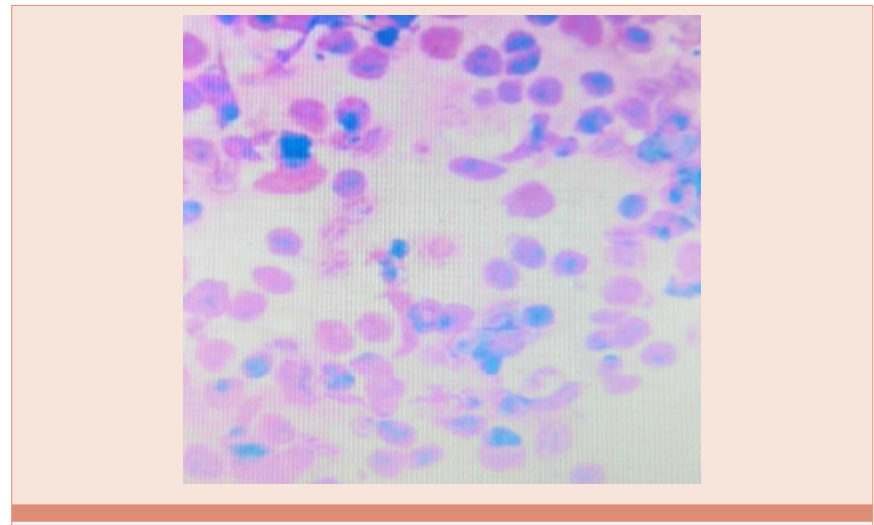

Figure 4: FNA Smear of Peri-portal Lymph Node.
Obstructive jaundice secondary to tubercular lymphadenitis can be confused with hepatobiliary malignancy [6].

TB lymphadenitis can be suspected when a contrast-enhanced CT scan shows hypodense masses with peripheral enhancement or when ERCP shows a normal pancreatogram with a smooth narrowing of the CBD $[7,8]$. US or CT-guided percutaneous fine needle aspiration (FNA) of the enlarged lymph nodes may be useful [9]. Cytological examination of the CBD aspirate obtained by ERCP may show acid-fast bacillus on Ziehl-Neelsen staining or may be subjected to TB-PCR for confirmation [10]. Early preoperative diagnosis of abdominal tuberculosis causing obstructive jaundice allows a more conservative management and better outcome of the disease as was done in our case and the patient responded to the initiation of ATT with improvement in clinical status and serum bilirubin levels.

\section{Conclusion}

Though obstructive jaundice secondary to abdominal tubercular lymphadenitis is rare, it should be considered as a differential diagnosis in $\mathrm{TB}$ endemic area and early treatment can prove to be lifesaving to the patient.

\section{References}

1. Ray S, Chatterjee S, Saha AK, Samanta S (2012) Obstructive jaundice due to tuberculosis of distal $\mathrm{CBD}$ and periampullary region mimic cholangiocarcinoma. Niger J Surg 18: 17-18.

2. Colovic R, Grubor N, Jesic R, Micev M, Jovanovic T, et al. (2008) Tuberculous lymphadenitis as a cause of obstructive jaundice: a case report and literature review. World J Gastroenterol 14: 3098-3100.

3. Ko YW, Hwang EH, Lee DW, Rhim DI (1976) Obstructive jaundice due to tuberculous lymphadenitis and/or tuberculosis of the pancreas: a case report. Korean J Gastroenterol 8: 67-75.

4. Chong VH (2008) Hepatobiliary tuberculosis: a review of presentations and outcomes. South Med J 101: 356-361.

5. Colovic R, Grubor N, Jesic R, Micev M, Jovanovic T, Colovic N, et al. (2008) Tuberculous lymphadenitis as a cuase of obstructive jaundice: a case report and literature review. World J Gastroenterol 14: 3098-3100.

6. Alvarez SZ (1998) Hepatobiliary tuberculosis. J Gastroenterol Hepatol 13: 833-839.

7. Mathieu D, Ladeb MF, Guigui B, Rousseau M, Vasile N (1986) Periportal tuberculous adenitis: CT features. Radiology 161: 713-715.

8. Obama K, Kanai M, Taki Y, Nakamoto Y, Takabayashi A (2003) Tuberculous lymphadenitis as a cause of obstructive jaundice: report of a case. Surg Today 33: 229-231.

9. El Mansari O, Tajdine MT, Mikou I, Janati MI (2003) [Pancreatic tuberculosis. Report of two cases] Gastroenterol Clin Biol 27: 548-550.

10. Probst A, Schmidbaur W, Jechart G, Hammond A, Zentner J, et al. (2004) Obstructive jaundice in AIDS: diagnosis of biliary tuberculosis by ERCP. Gastrointest Endosc 60: 145-148. 